\title{
Looking Back at Fetal Medicine in India in 2018, and Looking Forward to 2019
}

\author{
Ishwar Verma $^{1} \cdot$ Meenakshi Lallar $^{1} \cdot$ Veronica Arora $^{1}$
}

Published online: 4 June 2019

(C) Society of Fetal Medicine 2019

The year gone by has been a great year for the Society of Fetal Medicine. Thirty conferences on various aspects of fetal medicine were held in cities such as Delhi, Mumbai, Kolkata, Hyderabad, Bangalore, Nagpur, Raipur, Amritsar, Ludhiana, Patiala, Kochi, Visakhapatnam, Trichy, Vijayawada, Bhubaneswar, Cuttack, etc. My favorite was at Corbett Retreat among the tigers. What a great place to meet and learn. Ten conferences have already been held from January to March 15, 2019, and 12 conferences are lined up till Dec 2019. All credit to the President Dr Ashok Khurana, the Secretary Dr. Vivek Kashyap, and the executive committee for their excellent performance.

The journal also came out very well, with increasing the number of published pages in each issue and improving the quality of printing of text as well as images, thanks to the personal interest taken by Dr. Khurana. Our members, who number 2200, have extensively participated in the conferences organized by the Society and leading to an improvement in the quality of the ultrasonographic reports on fetal status, and eventually better feto-maternal care. Now the time has come for the members to document their efforts and write and publish the interesting cases seen by them, or compiling educative series for publication, to provide an outlet for their academic pursuits and to help other fetal medicine specialists manage similar cases.

The year 2018 saw a marginal expansion of the NIPT (Non-invasive prenatal testing) market. The test is markedly underutilized, firstly due to the restrictions (rightly) placed by the PNDT Act (Prenatal Diagnostic Techniques

Ishwar Verma

jfm200@yahoo.com

1 Institute of Medical Genetics and Genomics, Sir Ganga Ram Hospital, Rajinder Nagar, New Delhi 110060, India
Act), and secondly, the obsession of the Indian patients to aim at $100 \%$ exclusion of chromosomal disease. In most parts of the world $99.7 \%$ exclusion (sensitivity of NIPT) is good enough for the patients, but not in India. The fetal medicine specialists need to explain at great length that an exclusion of this magnitude $(99.7 \%)$ is adequate and acceptable. The publication of the landmark study in India on use of NIPT demonstrated that we in India could do a multicentric study as well as elsewhere, and the result of analysis was at par with studies from outside India [1]. We also learnt to avoid NIPT in cases of previous miscarriages or presence of children with intellectual disability or genetic disorders in the family, as invasive testing would be preferred under such circumstances. More-over, in families belonging to peripheral areas invasive testing offers a once-for-all test as opposed to NIPT which may result in recall/s either for confirmatory testing or because of inadequate fetal fraction. We learnt that the positive predictive value of NIPT is less for the sex chromosome disorders, emphasizing year again that confirmatory testing in NIPT is essential, as it is still a screening test. Microdeletions as well as abnormalities of other chromosomes being diagnosed by NIPT technique have not appealed much to Indian experts or patients because of their low incidence and low positive predictive value. We had believed that one of the major benefits of the NIPT technology would be its application in peripheral areas to replace amniocentesis/chorionic villus sampling and restricting the number of patients who genuinely require invasive procedures. However, this has not happened. Perhaps the vendors should examine the reason for this and improve the uptake of NIPT in the peripheral areas. One reason is probably the lack of salespersons as well as obstetricians with adequate understanding of NIPT in the peripheral cities. Another important reason is the cost of 
NIPT testing, which is still exorbitant for Indians, especially in the absence of its confirmatory nature. Another extension of non-invasive testing is the diagnosis of single gene disorders through cffDNA. This would be immensely useful, however even in cases where the paternal mutation is different from the one the mother has, this has not been offered to any great degree, because the technology is still not fully validated. May be that is the revolution waiting to happen after the NIPT takes off to a greater degree and reaches grass root levels. That is also the reason why we have not witnessed the establishment of the non-invasive test to diagnose RH D status of the fetus in cases of Rh D immunization in India, although this would save a huge amount of money being spent upon RH D antibody injections in women who are $\mathrm{Rh}$ negative with a $\mathrm{Rh}-$ ve fetus [2].

However, it is heartening to see the increased use of chromosomal microarray by fetal medicine specialists in India, whether in cases of increased nuchal thickness, structural fetal abnormalities or for study of products of conception in cases of recurrent miscarriages. It has been shown that microarrays reveal an extra $6 \%$ of cases with copy number variation (CNV) after excluding those diagnosed by a standard karyotype, in case of fetal malformations [3]. There is still an issue with the reporting of variants of uncertain significance (VOUS). Therefore, one message we would like to pass along is that any VOUS, whether a $\mathrm{CNV}$ or a single base change, should not be taken on its face value and certainly not be mentioned to the patient or used for prenatal diagnosis unless one is sure of its pathogenicity.

This also reminds us to emphasize that the practice of advising invasive testing in mothers with advanced maternal age, only if the biochemical screening shows high risk for chromosomal disease is not appropriate. The reason is that the biochemical analytes even if in the low risk range leaves a residual risk of autosomal aneuploidies and performs even poorer in picking out those with high risk for sex chromosomal aneuploidies. Many of us in practice use a cut off of 38 years beyond which we advise invasive testing or NIPT.

There is much talk about ethnic and expanded carrier screening in the West as well India $[4,5]$. Carrier screening for diseases started as a policy with screening of he Ashkenazi Jews for Tay Sach disease. With the realization that the Ashkenazi Jews (AJ) are at high risk for many other genetic disorders the screening was extended to other disorders too, but it was all targeted screening for the mutations common among them. Subsequently carrier screening was extended to genetic mutations that are common in other ethnic groups to develop a pan-ethnic test [6-8]. Use of NGS (Next-Generation-Sequencing) technology for carrier screening is necessary in India, because knowledge of mutations in the various genes is inadequate. Use of NGS for carrier screening has been labelled as expanded carrier screening. It has been much talked about in health circles in India to reduce the burden of genetic disorders. It is a laudable program, but nobody talks about who would pay the charge for the test. The rich of course would be willing to pay, but they should be counselled and provided the information regarding the availability of this test as well as its various implications. If this has to be taken up as a national policy the state must make funds available for this purpose.

Two very interesting studies have recently been published on exome sequencing in fetuses with congenital malformations. The one from UK-The PAGE Consortium was carried out by the Wellcome Trust-Cambridge University collaboration [9]. The doctors and the parents were told they would not get the results for use in the current pregnancy. In this study a cohort of 610 fetuses with ultra-sono-graphically detected anomalies were evaluated. Pathogenic variants were observed in $8.5 \%$, while another $3.9 \%$ revealed possibly pathogenic variants (a total of $12.4 \%$ ). Diagnostic genetic variants were present in $15.4 \%$ of skeletal anomalies, $15.4 \%$ of fetuses with multisystem anomalies (i.e. more than one fetal structural anomaly), $11.1 \%$ of fetuses with cardiac anomalies, and $9.2 \%$ in hydrops. However, diagnostic genetic variants were least common in fetuses with isolated increased nuchal translucency $(\geq 4.0 \mathrm{~mm})$ in the first trimester, and in those with facial, brain, renal and chest anomalies (3\% or less). In another study by Petrovski and colleagues from Colombia (NY) the results were made available to the doctors and the patients [10]. This study screened consecutive fetuses with structural anomalies from 2015 to 2017 and performed WES on 234 fetus-parent trios. Pathogenic variants were detected in $10.3 \%$ of fetuses. The maximum number detected were as follows: skeletal dysplasias (24\%), CNS (22\%), lymphatic effusions (24\%, renal (16\%) and IUGR (10\%). These observations suggest that in cases of fetal anomalies in which assessment with karyotype testing and chromosomal microarray fail to determine the underlying cause of a structural anomaly (ies), WES can add clinically relevant information that could assist in management of a pregnancy. These results are indeed promising and provided one can shorten the time for the study and analysis, this technology would be increasingly adopted in practice [11].

From cutting edge ultrasound technologies and molecular diagnostics we have indeed come a far way in fetal diagnostics. So, plausibly the next step should be advancement in fetal therapy [12]. And it is indeed quite pleasing to see that in India some centers of excellence in fetal medicine are already offering standard of care treatment in complicated monochorionic twin pregnancies like 
fetoscopic laser photocoagulation and radiofrequency ablation. Amniotic band resection and fetal shunt placement procedures (pleuro-amniotic and vesico-amniotic) are also being done with success. The multi-disciplinary collaboration involved in fetal ex-utero intrapartum treatment (EXIT) procedures, that have been successfully done is truly commendable. Some centers have even performed fetal balloon aortic valvuloplasty and lower urinary tract obstruction ablation via lasers. Overall, it is heartening to see that we are not very far behind the developed world in fetal therapeutics and with the dedication and expertise of our specialists we will continue to tread along the path of success in fetal therapy. However, as fetal therapeutic procedures are being done at limited centers where the expertise is available it is imperative that a close watch on fetal outcomes and long term follow up of these cases is maintained via meticulous record keeping and follow up so as to know the success of fetal intervention and final outcomes. Starting a registry for this purpose would be appropriate. Research publications from such centers will help guide fetal therapy challenges and outcomes in the Indian scenario.

At the global level this year saw some breakthroughs in fetal therapy. There has been much progress regarding fetal surgery for meningomyelocele. Recently a 30-month postnatal study of "management of meningomyelocele study (MOMS) has been published [13]. It was shown that for every two fetuses operated one will not require a shunt, and for every 5 fetuses operated one additional baby will be able to walk. The long-term outcomes are also improving. In addition, there have been attempts at carrying out the surgery by fetoscopy avoiding the hazards of open uterine surgery. However keeping in mind the current attitude of Indian patients one cannot see much future in surgery for meningoceles in India, because the burden of having a child with disabilities is still too high and the facilities provide by the government too inadequate, so that parents would prefer termination of a fetus with myelomeningocele rather than subject it to fetal surgery.

A group at Johns Hopkins successfully treated a cardiac rhabdomyoma causing outlet obstruction and supra-ventricular tachycardia by maternal administration of sirolimus, leading to shrinkage of the tumor [14]. The drug has been used as an inhibitor of m-TOR pathway for the treatment of large tumors in tuberous sclerosis, but this was the first use of it in fetal life. Even more fascinating was the treatment of twins with $\mathrm{x}$-linked form of ectodermal dysplasia with injection of a recombinant protein, which contained $\mathrm{IgG} 1$ and receptor binding portion of ectodysplasin [15]. In this disorder a pathogenic variant in EDA gene leads to decreased or absent sweating, abnormal nails, teeth and hair. Understanding of the pathophysiology of the disease and after trying injection of the recombinant protein in mice the same was injected in three fetuses carrying the mutation in EDA gene. The result was a near normal number of sweat glands and more tooth germs at birth as compared with untreated siblings. Not only did they discover intrauterine therapy but also a method for prenatal diagnosis of the disorder by observing the number of tooth germs in the mandible. It is likely that this technology can be used to treat many other disorders after a proper understanding of the pathophysiology by supplying the end-product protein.

Many new treatments such as exon skipping for Duchenne muscular dystrophy, and exon inclusion (spinal muscular atrophy) using anti-sense oligonucleotides have been introduced in practice. In utero gene therapy [16] and CRISPER -caspase 9 editing system to alter the mutant alleles in utero [17] have been introduced in 2018 and will find greater use in the coming years. For example, large deletions in $\gamma-\beta$ globin genes lead to increase in fetal $\mathrm{Hb}$, which mitigates the clinical severity of sickle cell disease and $\beta$-thalassemia. Antoniani et al. [18] designed a CRISPR/Cas9 strategy to disrupt a $13.6-\mathrm{kb}$ genomic region encompassing the $\delta$ - and $\beta$-globin genes and a putative $\gamma-\delta$ intergenic fetal hemoglobin ( $\mathrm{HbF})$ silencer. This caused a modest increase in fetal hemoglobin thus ameliorating the phenotype of thalassemia and sickle cell anemia. This has the potential to provide therapy for these two disorders. It is said that the best results of treatment are obtained if it is started early in the disease process, and there is nothing earlier than fetal life. After the discovery of CRISPR it is now being said, "if it can be cut, it can be cured." We can look forward to exciting milestones ahead giving the couples the joy of having a normal baby in place of one with abnormalities.

\section{References}

1. Verma IC, Puri R, Venkataswamy E, Tayal T, Nampoorthiri S, Andrew C, et al. Single nucleotide polymorphism-based noninvasive prenatal testing: experience in India. J Obstet Gynecol India. 2018;68(6):462-70. https://doi.org/10.1007/s13224-0171061-9.

2. Verma IC. Noninvasive prenatal testing: the Indian perspective. J Fetal Med. 2014;1:113-8. https://doi.org/10.1007/s40556-0140025-8).

3. Stosic M, Levy B, Wapner R. The use of chromosomal microarray analysis in prenatal diagnosis. Obstet Gynecol Clin N Am. 2018;45:55-68. https://doi.org/10.1016/j.ogc.2017.10.002.

4. King JR, Klugman S. Ethnicity-based carrier screening. Obstet Gynecol Clin N Am. 2018;45:83-101. https://doi.org/10.1016/j. ogc.2017.10.010.

5. Gregg AG. Expanded carrier screening. Obstet Gynecol Clin N Am. 2018;45:103-12. https://doi.org/10.1016/j.ogc.2017.10.005.

6. Ghidini A, Bianchi DW, Levy B, Mieghem TV, Deprest J, Chitty LS. In case you missed it: the prenatal diagnosis editors bring you 
the most significant advances of 2018. Prenat Diagn. 2019;39:61-9. https://doi.org/10.1002/pd.5407.

7. Johansen Taber KA, Beauchamp KA, Lazarin GA, Muzzey D, Arjunan A, Goldberg JD. Clinical utility of expanded carrier screening: results guided actionability and outcomes. Genet Med. 2018. https://doi.org/10.1038/s41436-018-0321-0 (Epub ahead of print).

8. Punj S, Akkari Y, Huang J, et al. Preconception carrier screening by genome sequencing: results from the clinical laboratory. Am J Hum Genet. 2018;102(6):1078-89.

9. Lord J, McMullan DJ, Eberhardt RY, et al. Prenatal exome sequencing analysis in fetal structural anomalies detected by ultrasonography (PAGE): a cohort study. Lancet. 2019. https:// doi.org/10.1016/S0140-6736(18)31940-8.

10. Petrovski S, Aggarwal V, Giordano JL, et al. Whole-exome sequencing in the evaluation of fetal structural anomalies: a prospective cohort study. Lancet. 2019. https://doi.org/10.1016/ S0140-6736(18)32042-7.

11. Normand EA, Alaimo JT, Van den Veyver IB. Exome and genome sequencing in reproductive medicine. Fertil Steril. 2018;109(2):213-20. https://doi.org/10.1016/j.fertnstert.2017.12. 010 (Epub 2018 Feb 1).
12. Baumgarten HD, Flake AW. Fetal surgery. Pediatr Clin N Am. 2019;66:295-308. https://doi.org/10.1016/j.pcl.2018.12.001.

13. Farmer DL, Thom EA, Brock JW 3rd, et al. The management of myelomeningocele study: full cohort 30-month pediatric outcomes. Am J Obstet Gynecol. 2018;218(2):256.e1-13.

14. Barnes BT, Procaccini D, Crino J, et al. Maternal sirolimus therapy for fetal cardiac rhabdomyomas. $N$ Engl J Med. 2018;378(19):1844-5.

15. Hammersen J, Wohlfart S, Goecke TW, et al. Reliability of prenatal detection of X-linked hypohidrotic ectodermal dysplasia by tooth germ sonography. Prenat Diagn. 2018. https://doi.org/10. 1002/pd.5384.

16. MacKenzie TC. Future avenues for in utero gene therapy. Cell Stem Cell. 2018;23(3):320-1.

17. Rossidis AC, Stratigis JD, Chadwick AC, Hartman HA, Ahn NJ, $\mathrm{Li} \mathrm{H}$, et al. In utero CRISPR-mediated therapeutic editing of metabolic genes. Nat Med. 2018;24(10):1513-8. https://doi.org/ 10.1038/s41591-018-0184-6 (Epub 2018 Oct 8).

18. Antoniani C, Meneghini V, Lattanzi A, Felix T, Romano O, Magrin E, et al. Induction of fetal hemoglobin synthesis by CRISPR/Cas9-mediated editing of the human b-globin locus. Blood. 2018;131(17):1960-73. 